\title{
Hepatocellular carcinoma presenting as thoracic spinal canal metastasis with no clinical primary foci: A report of a rare case and review of the literature
}

\author{
RUNZHE CHEN $^{1}$, ZENGXIN GAO $^{2}$, XIAOTAO WU ${ }^{2}$, JOS L. CAMPBELL $^{3}$, PEI ZHANG ${ }^{2}$ and BAOAN CHEN ${ }^{1}$ \\ Departments of ${ }^{1}$ Hematology and Oncology, and ${ }^{2}$ Spine Surgery, Zhongda Hospital, Medical School, \\ Southeast University, Nanjing, Jiangsu 210009, P.R. China; ${ }^{3}$ Department of Radiology, \\ School of Medicine, Stanford University, Stanford, CA 94305, USA
}

Received October 9, 2014; Accepted July 7, 2015

DOI: $10.3892 / 01.2015 .3569$

\begin{abstract}
Hepatocellular carcinoma (HCC) is the third most common malignant tumor worldwide and has a poor survival rate. The poor prognosis can be attributed to several of the characteristics of HCC, such as fast infiltrating growth, early-stage metastasis, high-grade malignancy and poor therapeutic efficacy. The current study presents a case of HCC that was metastatic to the spinal canal with an unknown primary site and discusses the diagnostic probabilities. The patient was a 48-year-old female who presented with chest paraesthesis of the back and numbness of the right lower limb. Computed tomography (CT) and magnetic resonance imaging indicated a possible lipomyoma in the thoracic spinal canal. Surgery was performed to remove the mass and the post-operative pathological diagnosis indicated a moderately-differentiated HCC. Subsequent abdominal CT scans and B-mode ultrasound failed to localize the primary foci in the liver and the tumor markers were normal. The patient had no history of chronic liver disease in the past. The patient refused any further examinations after surgery and was discharged from hospital. A post-operative follow-up 1.5 years later found that the patient was healthy and that the level of discomfort had been significantly reduced following the surgery. HCC presenting as thoracic spinal canal metastasis with an unknown primary site is extremely rare. The present study additionally reports the results of a literature
\end{abstract}

Correspondence to: Dr Baoan Chen, Department of Hematology and Oncology, Zhongda Hospital, Medical School, Southeast University, 87 Dingjiaqiao, Gulou, Nanjing, Jiangsu 210009, P.R. China

E-mail: cba8888@hotmail.com

Dr Pei Zhang, Department of Spine Surgery, Zhongda Hospital, Medical School, Southeast University, 87 Dingjiaqiao Nanjing, Jiangsu 210009, P.R. China

Email: zhangpeiseu@163.com

Key words: hepatocellular carcinoma, metastasis, primary foci review and provides a rational deduction for the unknown primary foci of HCC.

\section{Introduction}

Hepatocellular carcinoma (HCC) is the third most common malignant tumor worldwide, accounting for $6 \%$ of all tumors, with a five-year survival rate of $\sim 10 \%(1,2)$. HCC has a number of characteristics that this poor survival rate may be attributed to, including fast infiltrating growth, high-grade malignancy, early-stage metastasis and a poor therapeutic efficacy. At present, no optimal screening modalities have been established and HCC is monitored by abdominal ultrasound examinations every 6 months, despite this technique exhibiting inadequate sensitivity. The optimum treatment for HCC is orthotopic liver transplantation (OLT), however, other treatment modalities are also available, which include surgery (resection and liver transplantation), radiological techniques, systemic chemotherapy and molecularly targeted therapies (3). Numerous HCC patients succumb to their condition quickly due to the rapid tumor progression and metastasis (4). HCC can metastasize to the lungs, bones, adrenal glands, brain and other organs and tissues. However, even in patients with advanced unresectable HCC, the rate of extrahepatic metastasis is limited (5). According to previous studies, a primary site is not established in $2-5 \%$ of all newly diagnosed cancer patients with metastases, even following a complete diagnostic work-up (6). To date, there have been no studies on HCC metastases in which no primary foci can be found. Therefore, the current study presents a case of liver carcinoma metastatic to the thoracic duct with an unknown primary site and discusses the relevant diagnostic probabilities.

\section{Case report}

A 48-year-old female was admitted to the Department of Spine Surgery (Zhongda Hospital, Southeast University, Nanjing, Jiangsu, China) in December 2011, presenting with chest paraesthesis of the back and numbness of the right lower limb. Walking distance was also shortened significantly compared with that of the previous 6 months. A physical examination 


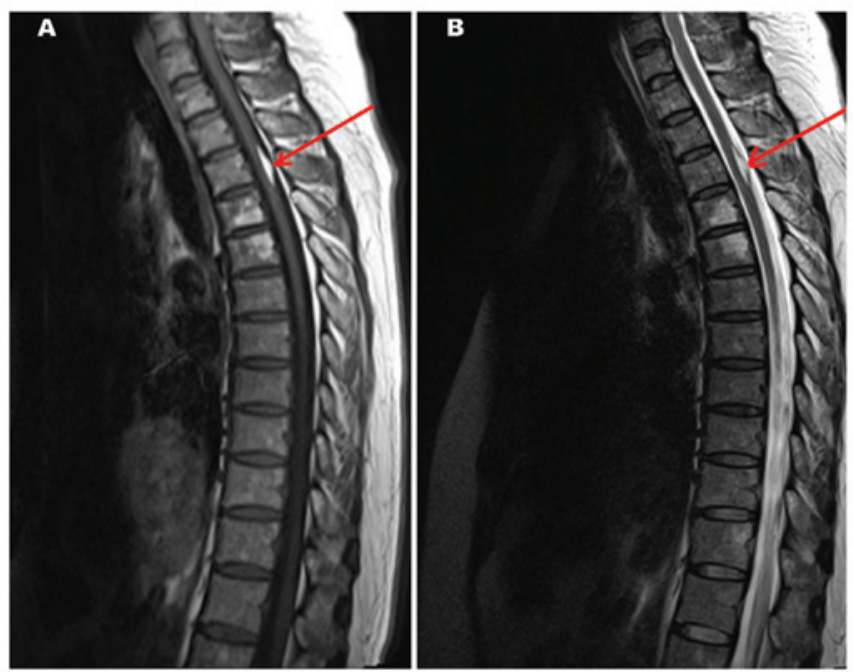

Figure 1. Magnetic resonance imaging showing a subdural extramedullary high-intensity signal at the T3 level. (A) T1- and (B) T2-weighted images Arrows indicate the high-intensity signal at the $\mathrm{T} 3$ level.

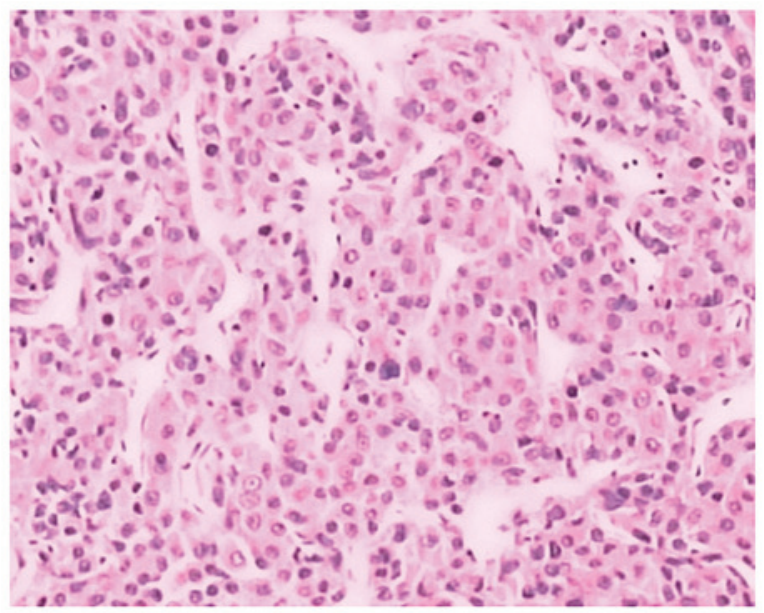

Figure 2. Histopathological examination of the mass revealing moderately-differentiated hepatocellular carcinoma. Hematoxylin and eosin staining. Magnification, x100.

showed nothing wrong, with the exception that the bilateral knee jerk and Achilles tendon reflex were active. Upon admission, magnetic resonance imaging (MRI) of the thoracic spine and cervical spine indicated the degeneration of the cervical vertebrae, mild protrusion of intervertebral discs at $\mathrm{C} 3 / 4,5 / 6$ and 6/7, and a subdural extramedullary high-intensity signal at the T3 level that was highly suspected to be a lipoma (Fig. 1). Computed tomography (CT) scans and two-dimensional reconstruction also indicated a low intensity signal in the spinal canal at the T3 level and strengthened the probability of a lipoma diagnosis.

The patient received posterior surgery via a intramedullary tumor resection at the T3 vertebra, internal fixation of screws at the T2-4 pedicle, bone graft fusion between the transverse processes and intradural exploration. The spinal cord was expanded and during the exploration, a yellowish soft mass was found in the T3 spinal canal. The mass was $\sim 4 \times 4 \times 20 \mathrm{~mm}$ and was well defined. The mass was isolated from the spine
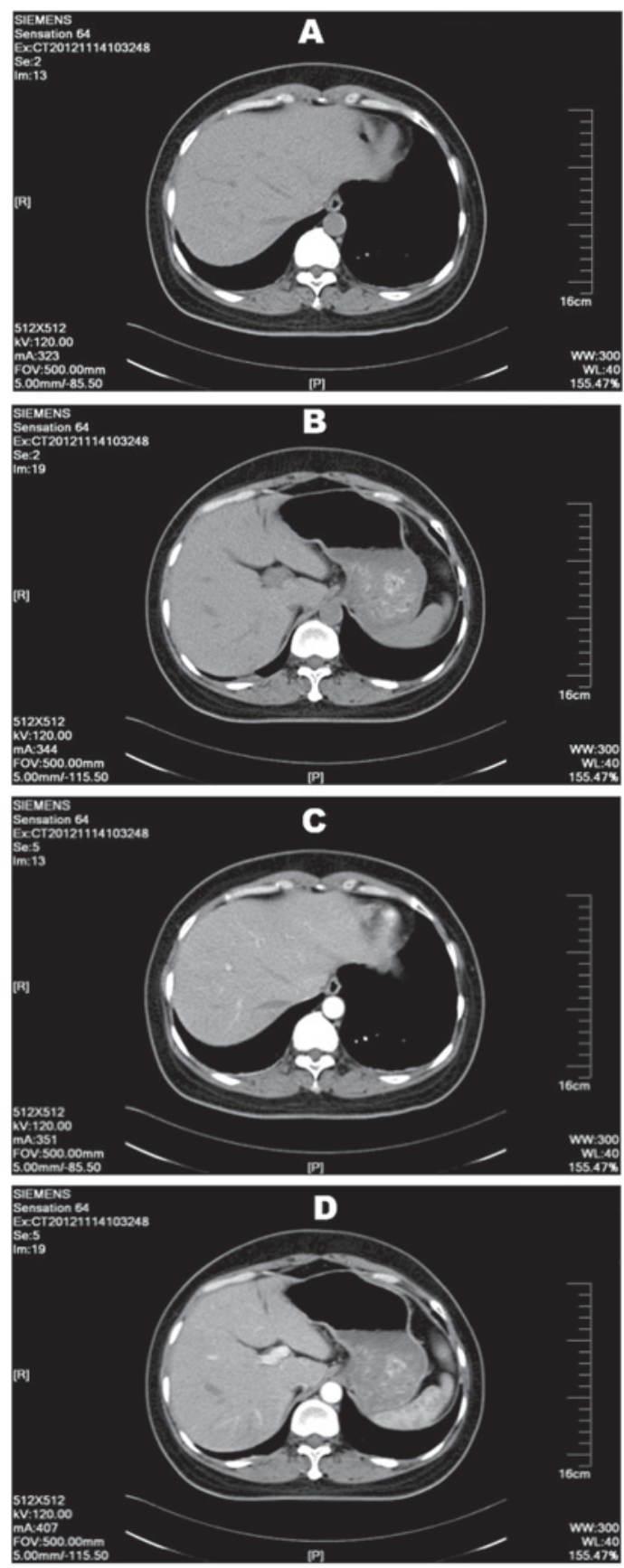

Figure 3. Upper abdominal plain and enhanced CT scans revealing a slightly lower density mass in the right hepatic lobe that was not well defined, with a size of $\sim 9 \times 7 \mathrm{~mm}$. Plain CT at (A) $13 \mathrm{~mm}$ and (B) $19 \mathrm{~mm}$ levels; enhanced CT at (C) $13 \mathrm{~mm}$ and (D) $19 \mathrm{~mm}$ levels. CT, computed tomography.

gradually until no tissue residue remained and no clear hemorrhage from the wound surface was left. Unexpectedly, the post-operative histopathological examination revealed a moderately-differentiated HCC (Fig. 2). Immunohistological examination results were as follows: Cytokeratin (CK)19(-), Hep1(+), glypican-3(+), cluster of differentiation (CD)10(+), CK7(-), synaptophysin(-), chromogranin A(-), IMP-3(-) and CD56(-), with a Ki-67 of $~ 6 \%$, which was in accordance with the diagnosis of HCC. As the pathological diagnosis was not in agreement with the imaging diagnosis and intraoperative exploration, the pathologist suggested further examinations of the liver. 
Subsequent examinations were performed to yield further information to base the developing diagnosis on. Several tumor markers were negative, including $\alpha$-fetoprotein (AFP), carcinoembryonic antigen, cancer antigen (CA)19-9, CA12-5 and CA15-3. These tumor markers were measured again 1 month later and were again found to be negative. Tests for the antigens and antibodies of hepatitis $\mathrm{B}$ and $\mathrm{C}$ were all negative. Abdominal B-mode ultrasound indicated that the liver was normal and no abnormal signals were observed. Examinations after 3 months and again after 12 months by abdominal B-mode ultrasound showed no subsequent anomalies. Upper abdominal plain and enhanced CT scans revealed a slightly lower density mass in the right hepatic lobe that was not well defined. The mass was $\sim 9 \times 7 \mathrm{~mm}$, and as the only anomaly within the liver, this site was suspected as the primary liver hemangioma (Fig. 3), however, no positive diagnosis could be made. The patient had no history of chronic liver disease in the past.

The post-operative neurological symptoms improved greatly and the chest pain was relieved significantly. The patient refused any further examinations regarding the disease and was discharged from the hospital. A post-operative follow-up examination was performed at 3 months, 6 months, 1 year and 1.5 years. The chest paraesthesis and numbness of the right lower limb were greatly relieved and there were no persistent physiological symptoms. Written informed consent was obtained from the patient prior to the publication of the data.

\section{Discussion}

HCC presenting as thoracic duct metastasis with an unknown primary site is extremely rare. In the present study, the patient exhibited no typical clinical presenting symptoms associated with HCC, such as liver pain, fatigue, jaundice, palmar erythema or gynecomastia. Early-stage HCC can be asymptomatic or patients can only have mild cancer-related symptoms (7). Once a patient presents with liver carcinoma-related symptoms, it is almost certain that the individual is in the intermediate or advanced stages of the disease. A number of techniques are available for the treatment of intermediate-stage disease, including surgery, transarterial chemoembolization, radioembolization and radiofrequency ablation (8).

Given the clinical presentation and imaging results of the patient in the present study, lipomyoma was the most likely diagnosis. During surgery, a yellowish soft mass was found and removed from the spinal canal. This was believed to be the mass indicated by the MRI in Fig. 1; unfortunately no sample was preserved after removal. Unexpectedly, the immediate histopathological examination revealed a moderately-differentiated HCC. Pathological diagnosis is considered as the gold standard diagnostic technique, however, the results were not in accordance with the previous imaging diagnosis and intraoperative exploration findings. The pathology results were rechecked three times, yet each time the results pointed to an HCC. The immunohistochemical markers indicated that it was moderately-differentiated, yet subsequent laboratory examinations indicated almost nothing abnormal in the patient's liver. CT was the only modality that indicated a small area of slightly lower density in the right hepatic lobe, measuring $\sim 9 \times 7 \mathrm{~mm}$. This region was highly suspicious, however, tumor markers were negative and abdominal ultrasound suggested normal tissue. The patient continued to undergo abdominal ultrasound scans every 6 months without any indication of anomalies. Given that ultrasound is an extremely sensitive indicator for liver carcinoma, this led us to conclude that no primary site within the liver could be located that agreed with the pathology results. According to the CT results and the pathological examination, we have several hypotheses about the primary site. Firstly, in the embryonic period of the patient, the gene-mutated liver cancer cells may have metastasized or been implanted in the spinal canal, and over time the tumor mass would have compressed the spinal canal and the patient would begin to express symptoms. Secondly, the low-density mass in the liver found by CT could have been the primary foci, but due to the small size, examinations may have failed to recognize that it was a tumor mass. It is also possible that the size of the primary tumor affected the tumor marker test, as the AFP sensitivity decreases from 52 to $25 \%$ as the tumor diameter decreases below $3 \mathrm{~cm}$ (9).

According to previous studies, metastatic disease occurs in approximately one-third of all advanced cancers and the primary site of origin is initially uncertain in a number of these patients. Cancer registries around the world report the incidence of cancer of unknown primary (CUP) in the range of $2-10 \%$ of all cancer diagnoses $(10,11)$. Furthermore, CUP is among the top 10 most common malignancies (11). According to large postmortem cohort studies, the most common primary sites of CUP include the lungs $(27 \%)$, pancreas (24\%), liver or bile ducts (8\%) (12). The majority of studies on tumors of unknown primary site are of malignant melanoma, yet reports of liver carcinoma with no primary foci are seldom mentioned. Recently, Boussios et al (13) reported a similar case of neuroendocrine carcinoma of unknown primary site, however, in contrast to the current study, the patient received palliative radiation to relieve local symptoms and achieved a partial response. The follow-up examinations in the present study indicated that the patient lived healthily and exhibited no liver carcinoma-associated symptoms post-surgery.

To the best of our knowledge, this is the first study to report a liver carcinoma patient who had thoracic duct metastasis with an unknown liver primary site and no associated clinical symptoms. All the examinations performed suggested that there was almost nothing abnormal with the patient's liver. Yet the pathology strongly suggested the presence of liver carcinoma, while ultrasound and tumor markers were negative. CT suggested an extremely small area of decreased signal in the right hepatic lobe, but the diagnosis may have been hampered by the small size of the possible primary foci. Extensive post-surgery examinations over 1.5 years showed no change in the liver and no presentation of associated symptoms. Due to its rarity, this case has been documented to the best of our abilities.

\section{Acknowledgements}

The authors would like to thank the reviewers and editors for their contributions to improving the quality of the manuscript. The study was supported by the National Natural 
Science Foundation of China (grant nos. 81170492 and 81370673), the Key Medical Projects of Jiangsu Province (grant no. BL2014078) Key Discipline of Jiangsu Medicine (2011-2015) and, in part, by the Victorian Government of Australia in the form of a Victorian Postdoctoral Research Fellowship.

\section{References}

1. Yang JD and Roberts LR: Hepatocellular carcinoma: A global view. Nat Rev Gastroenterol Hepatol 7: 449-458, 2010.

2. El-Serag HB: Epidemiology of viral hepatitis and hepatocellular carcinoma. Gastroenterology 142: 1264-1273, 2012.

3. Attwa MH and El-Etreby SA: Guide for diagnosis and treatment of hepatocellular carcinoma. World J Hepatol 7: 1632-1651, 2015.

4. Jain S, Singhal S, Lee P and Xu R: Molecular genetics of hepatocellular neoplasia. Am J Transl Res 2: 105-118, 2010.

5. Jarnagin WR: Management of small hepatocellular carcinoma: A review of transplantation, resection and ablation. Ann Surg Oncol 17: 1226-1233, 2010.

6. Petrakis D, Pentheroudakis G, Voulgaris E and Pavlidis N: Prognostication in cancer of unknown primary (CUP): Development of a prognostic algorithm in 311 cases and review of the literature. Cancer Treat Rev 39: 701-708, 2013.
7. Raza A and Sood GK: Hapatocellular carcinoma review: Current treatment, and evidence-based medicine. World J Gastroenterol 20: 4115-4127, 2014.

8. Bruix J, Gores GJ and Mazzaferro V: Hepatocellular carcinoma: Clinical frontiers and perspectives. Gut 63: 844-855, 2014.

9. Saffroy R, Pham P, Reffas M, Takka M, Lemoine A and Debuire B: New perspectives and strategy research biomarkers for hepatocellular carcinoma. Clin Chem Lab Med 45: 1169-1179, 2007.

10. Greco FA and Hainsworth JD: Cancer of unknown primary site. In: Cancer: Principles and Practice of Oncology. DeVita VT Jr, Hellman S and Rosenberg S (eds). 8th edition. Lippincott, Williams \& Wilkins, Philadelphia, PA, pp2363-2387, 2008.

11. Greco FA, Oien K, Erlander M, Osborne R, Varadhachary G, Bridgewater J, Cohen D and Wasan H: Cancer of unknown primary: Progress in the search for improved and rapid diagnosis leading toward superior patient outcomes. Ann Oncol 23: 298-304, 2012

12. Pentheroudakis G, Golfinopoulos V and Pavlidis N: Switching benchmarks in cancer of unknown primary: From autopsy to microarray. Eur J Cancer 43: 2026-2036, 2007.

13. Boussios S, Kostadima V, Batistatou A, et al: Neuroendocrine cell carcinoma of unknown primary arising in long standing history of multiple sclerosis. Case Rep Oncol Med 2015: 135976, 2015. 